Digital Object Identifier (DOI) 10.1007/s10231-004-0118-4

Luigi Accardi · Francesco Fidaleo

\title{
Entangled Markov chains ${ }^{\star}$
}

Received: September 23, 2003; in final form: December 10, 2003

Published online: October 1, 2004 - (C) Springer-Verlag 2004

Abstract. Motivated by the problem of finding a satisfactory quantum generalization of the classical random walks, we construct a new class of quantum Markov chains which are at the same time purely generated and uniquely determined by a corresponding classical Markov chain. We argue that this construction yields as a corollary, a solution to the problem of constructing quantum analogues of classical random walks which are "entangled" in a sense specified in the paper.

The formula giving the joint correlations of these quantum chains is obtained from the corresponding classical formula by replacing the usual matrix multiplication by Schur multiplication.

The connection between Schur multiplication and entanglement is clarified by showing that these quantum chains are the limits of vector states whose amplitudes, in a given basis (e.g. the computational basis of quantum information), are complex square roots of the joint probabilities of the corresponding classical chains. In particular, when restricted to the projectors on this basis, the quantum chain reduces to the classical one. In this sense we speak of entangled lifting, to the quantum case, of a classical Markov chain. Since random walks are particular Markov chains, our general construction also gives a solution to the problem that motivated our study.

In view of possible applications to quantum statistical mechanics too, we prove that the ergodic type of an entangled Markov chain with finite state space (thus excluding random walks) is completely determined by the corresponding ergodic type of the underlying classical chain.

Mathematics Subject Classification (2000). Primary 46L53, 60J99; Secondary 46L60, 60G50, 62B10

Key words. non commutative probability and statistics - quantum Markov processes applications of selfadjoint operator algebras to physics - quantum random walks - quantum information theory

\section{Introduction}

The impetuous development of quantum information has raised the problem of finding a satisfactory quantum generalization of the classical random walks. The

L. Accardi: Centro Interdisciplinare Vito Volterra, II Università di Roma "Tor Vergata", Via Columbia 2, 00133 Roma, Italy, e-mail: accardi @volterra mat . uniroma2 . it

F. Fidaleo: Dipartimento di Matematica, II Università di Roma "Tor Vergata", Via della Ricerca Scientifica 1, 00133 Roma, Italy, e-mail: fidaleo@axp.mat . uniroma2 . it

* Work partially supported by Istituto Nazionale Di Alta Matematica and European Union Research Training Network: "Quantum Probability with Applications to Physics, Information Theory and Biology". 
relevance of this problem for quantum information has been emphasized in the past three years, in a large number of papers. Several different solutions of this problem have been proposed and studied, see e.g. [7]-[9], [11]-[14], [24]-[34], [38]-[40]. However, these proposals introduce some features which are not quite satisfactory from the mathematical point of view.

First of all, these constructions are based on special models and a general mathematical definition of a "quantum random walk" seems to be lacking. Second, all these constructions are based on a quantum evolution, unitary in some cases, irreversible in others. Now, random walks are particular cases of Markov processes and it is well known that, while in the classical case a Markov evolution uniquely determines the law of the corresponding stochastic process, this is, in general, false in the quantum case. Finally, a desirable requirement for a quantum extension of a family of classical processes, is that there should exist a standard procedure to embed the original classical family into its quantum extension. In a satisfactory quantum generalization of the classical random walks, all these requirements should be precisely formulated and fulfilled. In the present paper we concentrate our attention on this mathematical problem and we refer to the above quoted literature for the motivations and the potential applications to quantum information.

The problem of a quantum generalization of classical random walks has a long history. The non-unitary implementability of the symmetric 1-dimensional random walk was proved in [3]. Recently, this result has been extended to the non-symmetric case by Meyer ([33]), who proved that a non-symmetric random walk is unitarily implementable if and only if it is a one-side shift (i.e. shift along a fixed direction), namely, it is a totally deterministic process.

Another proposal was advanced in the paper [6], whose starting point was the characterization of the classical random walks as Markov chains with the additional property that their transition operators are convex combinations of reversible dynamics and their inverses. The idea was to define a quantum random walk as a quantum Markov chain whose forward Markov operator has the above mentioned property. The structure theorem proved in [6] for these quantum random walks, showed that they are convex combinations of product states. In other words, they have zero degree of entanglement. This feature makes them unattractive for applications to quantum information where one deals with pure states with a high degree of entanglement (i.e. very far from being convex combinations of product states).

To meet the needs of quantum information, one would like that quantum random walks correspond to pure quantum Markov chains. In fact, we will require a condition which is slightly weaker but much easier to verify, that is the quantum Markov chain under consideration should be "purely generated" in the sense of Fannes, Nachtergaele and Werner, see [20]. This implies, in particular, that the state at each instant is pure, up to a boundary effect that becomes negligible in the strong clustering case (cf. Theorem 3.4).

In summary, we list requirements that should be fulfilled by any candidate definition of a quantum random walk:

(1) it should be a quantum Markov chain;

(2) it should be purely generated; 
(3) its restriction on at least one maximal Abelian subalgebra, should be a classical random walk;

(4) it should be uniquely determined, up to arbitrary phases, by its classical restriction.

The 4-th requirement is particularly stringent because we require a quantum Markov chain to be uniquely determined by a classical transition operator, while we know that even the quantum transition operator is usually not sufficient to determine the chain.

In the present paper we propose a construction which satisfies the above criteria, and is applicable to processes with finite or countable state space. In particular, our construction is applicable to random walks (in fact to arbitrary, even nonhomogeneous, Markov chains).

Different constructions of purely generated (resp. pure) quantum Markov chains have been considered in [19], and successively in [10]. However, the following two questions studied in this paper were not investigated, namely:

(i) how to construct a purely generated (resp. pure) quantum Markov chain uniquely determined, up to phases, by a pre-assigned classical Markov chain;

(ii) how to include random walks in this construction.

The mentioned papers $[10,19]$ restrict their attention to finitely correlated states. Any notion of a quantum random walk satisfying Conditions (1) and (3) of the four above listed ones, cannot lead, in the most interesting situations, to finitely correlated states. On the contrary, the theory originally developed in [1] is applicable to arbitrary (hyperfinite) $C^{*}$-algebras, and this fact plays an essential role in our construction.

In order to give an intuitive idea of the connection of our construction with entanglement, let us note that the key characteristic of entanglement is the superposition principle and the corresponding interpretation of the amplitudes as "complex square roots of probabilities". This suggests a naïve approach in which, given a classical Markov chain with finite state space $S,\left\{\left(\Pi_{i j}\right),\left(\pi_{i}\right)\right\}_{i, j \in S}$, made of a stochastic matrix $\Pi$ and an initial distribution for it described by a row vector $\pi$, one can associate to it the superposition

$$
\psi_{n}=\sum_{j_{0}, \ldots, j_{n}} \sqrt{\pi_{j_{0}}} \prod_{\alpha=0}^{n-1} \sqrt{\Pi_{j_{\alpha}, j_{\alpha+1}}}\left|e_{j_{0}}, \ldots, e_{j_{n}}\right\rangle,
$$

where

$$
\left|e_{j_{0}}, \ldots, e_{j_{n}}\right\rangle:=e_{j_{0}} \otimes \cdots \otimes e_{j_{n}},
$$

and the square roots in (1.1) are determined up to phases (cf. (2.10)). The matrix elements $\left\langle A \psi_{n}, \psi_{n}\right\rangle$ become asymptotically constant on elements $A$ localized in finite subsets of $\mathbb{N}$. Hence,

$$
\varphi(A):=\lim _{n}\left\langle A \psi_{n}, \psi_{n}\right\rangle
$$




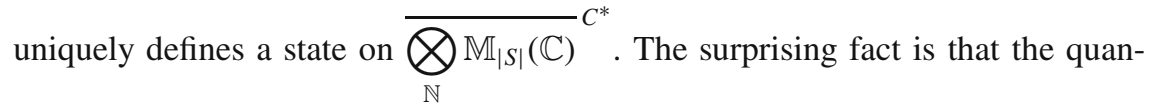
tum Markov chains, defined with this naïve approach can equivalently be defined abstractly in terms of the Schur multiplication. This gives a more algebraic representation of our construction which better illustrates why the above limit produces a quantum Markov chain.

The connection with entanglement can be intuitively understood by noticing that, for the identity of the Schur multiplication $E$,

$$
E=\sum_{i, j} e_{i j} \equiv \sum_{i, j}\left|e_{i}\right\rangle\left\langle e_{j}|=| \sum_{i} e_{i}\right\rangle\left\langle\sum_{j} e_{j}|=d| e\right\rangle\langle e|,
$$

where, in bra-ket notation,

$$
|e\rangle:=\frac{1}{\sqrt{d}} \sum_{j} e_{j}
$$

is precisely the maximally entangled unit vector in the basis $\left\{e_{j}\right\}$. Namely, the identity of the Schur multiplication is the projection on the maximally entangled vector in the chosen basis. The developments in the following sections will substantiate this intuition.

The present paper is organized as follows. Section 2 is devoted to defining an entangled lifting of classical Markov chains by the use of the Schur multiplication (called entangled Markov chains in the following). Then, the basic properties of this quantum lifting are investigated. As the Schur multiplication extends to a completely positive normal map of $\mathbb{M}_{\infty}(\mathbb{C}) \bar{\otimes} \mathbb{M}_{\infty}(\mathbb{C})$ onto $\mathbb{M}_{\infty}(\mathbb{C})$ (cf. [21]), most of our analysis applies mutatis mutandis to Markov chains with countably infinite state space. ${ }^{1}$ It is then to be hoped that our construction includes nontrivial examples of quantum random walks. Section 3 is devoted to a detailed analysis of the ergodic properties of the entangled Markov chains. Namely, it is shown that the ergodic type of an entangled Markov chain with finite state space is completely determined by the corresponding ergodic type of the underlying classical chain (cf. Theorems 3.3 and 3.4). The last results are not simple consequences of the theory of completely ergodic decomposition of quantum Markov chains developed in [20], whereas the pureness condition for entangled Markov chains follows from one of the pureness criteria of Theorem 1.5 of [20]. This is explained in some detail in Section 4, the last including some non-technical comments on entangled Markov chains. In view of the possible extensions to multi-dimensional examples, Section 5 is devoted to the non-homogeneous case. The paper is complemented with an appendix collecting standard properties of stochastic matrices repeatedly used in the following.

We end the introduction by noticing that the quantum Markov chains treated in the present paper could also have natural applications in quantum statistical

1 Equivalently, the transition operators of the entangled Markov chains under consideration are generated by isometries, see Lemma 2.2 (i.e. they are purely generated). Hence, the transition operators also make sense as maps of $\mathbb{M}_{\infty}(\mathbb{C}) \bar{\otimes} \mathbb{M}_{\infty}(\mathbb{C})$ onto $\mathbb{M}_{\infty}(\mathbb{C})$. 
mechanics. Indeed, when a translation invariant entangled (lifting of a classical) Markov chain is considered as a state on $\overline{\bigotimes_{\mathbb{Z}} \mathbb{M}_{d}(\mathbb{C})} C^{*}$, it leads to a convex combination of pure states which are again entangled Markov chains on regrouped algebras (Sections 3 and 4). Hence, due to the results in [19,35], entangled Markov chains lead to explicit examples of states on the one-dimensional spin chain which are ground states of suitable Hamiltonians.

\section{An entangled lifting of classical Markov chains}

Let $S$ be a finite set. A classical, not necessarily homogeneous, Markov chain with state space $S$ is uniquely determined by a pair

$$
\left\{\varphi_{0},\left(P_{n}\right)\right\},
$$

where:

(i) $\varphi_{0}$ is a state on $C(S)$, the algebra of all the complex-valued functions on $S$;

(ii) for each $n, P_{n}: C(S) \mapsto C(S)$ is a Markov operator.

The pair (2.1) uniquely determines the correlators

$$
\varphi_{0}\left(f_{0} \cdot P_{1}\left(f_{1} \cdot P_{2}\left(f_{2} \cdots P_{n}\left(f_{n}\right) \cdots\right)\right)\right),
$$

of the Markov chain, where $f_{k} \in C(S), k=0, \ldots, n$, and the dot denotes the pointwise multiplication in $C(S)$,

$$
(f \cdot g)(j)=f(j) g(j), \quad j \in S .
$$

It is known that there exists a unique state $\varphi_{c l}$ on the algebra

$$
C\left(\prod_{\mathbb{N}} S\right) \equiv \bar{\bigotimes}_{\mathbb{N}} C(S){ }^{*}
$$

such that the expectation value $\varphi_{c l}\left(f_{0} \otimes f_{1} \otimes \cdots \otimes f_{n} \otimes 1 \otimes \cdots\right)$ is given by (2.2). The main difference between classical and quantum Markov chains is due to the fact that the naïve generalization of Formula (2.2) to the case in which $C(S)$ is replaced by the matrix algebra $\mathbb{M}_{d}(\mathbb{C}), d:=|S|=$ cardinality of $S$, does not define a state because, contrarily to (2.3), the matrix multiplication

$$
A \otimes B \in \mathbb{M}_{d}(\mathbb{C}) \otimes \mathbb{M}_{d}(\mathbb{C}) \mapsto A B \in \mathbb{M}_{d}(\mathbb{C})
$$

is not positive. To recover positivity, it was proposed in [1] to replace the maps $A \otimes B \mapsto A P_{n}(B)$ by arbitrary completely positive, identity preserving maps

$$
\varepsilon_{n}: \mathbb{M}_{d}(\mathbb{C}) \otimes \mathbb{M}_{d}(\mathbb{C}) \mapsto \mathbb{M}_{d}(\mathbb{C}),
$$

called transition expectations. This leads, for $\left\{A_{0}, \ldots, A_{n}\right\} \subset \mathbb{M}_{d}(\mathbb{C})$, to the correlators

$$
\varphi_{0}\left(\mathcal{E}_{0}\left(A_{0} \otimes \mathcal{E}_{1}\left(A_{1} \otimes \cdots \otimes \mathcal{E}_{n}\left(A_{n} \otimes 1\right) \cdots\right)\right)\right)
$$


which are positive and projective, and hence define a unique state $\varphi$ on $\overline{\bigotimes_{\mathbb{N}} \mathbb{M}_{d}(\mathbb{C})} c^{*}$. Such a state is called a (generalized) quantum Markov chain.

Fixing a system $e \equiv\left\{e_{i j}\right\}_{i, j=1}^{d}$ of matrix units in $\mathbb{M}_{d}(\mathbb{C})$,

$$
e_{i j} e_{h k}=\delta_{j h} e_{i k}, \quad \sum_{i} e_{i i}=1, \quad i, j=1, \ldots, d,
$$

one obtains a natural embedding of $C(S)$ into $\mathbb{M}_{d}(\mathbb{C})$ given by

$$
f \in C(S) \mapsto \sum_{j=1}^{d} f(j) e_{j j} .
$$

Therefore, for any quantum Markov chain on $\overline{\bigotimes_{\mathbb{N}} \mathbb{M}_{d}(\mathbb{C})}{ }^{*}$, and for any system of matrix units $e$ in $\mathbb{M}_{d}(\mathbb{C})$, one obtains a classical probability measure on $\prod_{\mathbb{N}} S$. The family of all classical probability measures on $\prod_{\mathbb{N}} S$ obtained in this way is strictly larger than the family of classical Markov chains and it was fully characterized by Fannes, Nachtergaele and Slegers ([18]).

The natural generalization of the diagonal embedding (2.5), combined with a simple construction (see [2, Section 4]) leads to a class of Markov chains whose restriction to the $e$-diagonal algebra reproduces exactly all the classical Markov chains. However, the states obtained in this way are very near to classical states because they are defined by sequences of commuting density matrices, hence they have very poor "entanglement properties". In the present paper we propose another construction by replacing the diagonal embedding (2.5) by an "entangled embedding". The new ingredient which allows our construction to work, is to generalize the classical formula (2.2) by replacing the usual matrix multiplication by the Schur multiplication among matrices for which the map (2.4) is positive by Schur's lemma.

Consider a copy $\mathcal{M}_{j}$ of the algebra $\mathbb{M}:=\mathbb{M}_{d}(\mathbb{C})$ of all $d \times d$ matrices with complex entries, with $d$ kept fixed during the analysis. For each finite subset $F \subset \mathbb{Z}$, we put

$$
\mathfrak{M}_{F}:=\bigotimes_{j \in F} \mathcal{M}_{j}
$$

and, if $F \subset G$, we consider the natural embedding $a_{F} \mapsto a_{F} \otimes 1_{G \backslash F}$. The local algebra

$$
\mathfrak{M}:=\bar{\bigotimes}_{j \in \mathbb{Z}} \mathcal{M}_{j}{ }^{*}
$$

is the $C^{*}$-inductive limit associated to the directed system $\left\{\mathfrak{M}_{F}\right\}_{F \subset \mathbb{Z}}$, that is the UHF algebra. 
Let $\left\{e_{i j}\right\}_{i, j=1}^{d}$ be the canonical system of matrix units in $\mathbb{M}$. Denote $\mathbb{D}:=\mathbb{C}^{d} \subset$ $\mathbb{M}_{d}(\mathbb{C}) \equiv \mathbb{M}$ the corresponding diagonal algebra, where

$$
\left(a_{1}, \ldots, a_{d}\right) \mapsto \operatorname{diag}\left(a_{1}, \ldots, a_{d}\right)=\sum_{j=1}^{d} a_{j} e_{j j}
$$

In the following, we will denote with the same symbol an element in $\mathbb{C}^{d}$ and its image in $\mathbb{D}$. We denote by $\mathbb{E}: \mathbb{M} \mapsto \mathbb{D}$ the canonical Umegaki conditional expectation onto the diagonal algebra.

For our purpose, it is also of interest to consider the Abelian algebra

$$
\mathfrak{D}:=\bar{\bigotimes}_{j \in \mathbb{Z}} \mathfrak{D}_{j}{ }^{*},
$$

where $\mathscr{D}_{j}$ is for each $j \in \mathbb{Z}$, a copy of the diagonal algebra $\mathbb{D}$. We have $\mathfrak{D} \sim C(\Omega)$, the space of all continuous functions on the compact space $\Omega:=\prod_{j \in \mathbb{Z}} \Omega_{j}$ and $\Omega_{j}=$ $\{1, \ldots, d\}$ for all $j \in \mathbb{Z}$. Thus, $\mathfrak{D}$ is an Abelian subalgebra of $\mathfrak{M}$ in a canonical way. We denote by $\mathfrak{E}: \mathfrak{M} \mapsto \mathfrak{D}$ the canonical Umegaki conditional expectation of $\mathfrak{M}$ onto $\mathfrak{D}$ arising from the infinite tensor product of $\mathbb{E}$. We denote by $\tau$ the one-step shift on the chain. ${ }^{2}$ Finally, for general $C^{*}$-algebras $\mathfrak{A}, \mathfrak{B}$, a completely positive identity-preserving linear map $\mathcal{E}: \mathfrak{A} \otimes \mathfrak{B} \mapsto \mathfrak{B}$ sometimes will be called transition expectation.

To define the Schur multiplication, we choose an orthonormal basis $\left\{e_{j}\right\}_{j=1, \ldots, d}$ in a $d$-dimensional Hilbert space $\mathscr{H}_{d}$ which is kept fixed during the analysis. In such a way, we have the natural identification $\mathscr{H}_{d} \sim \mathbb{C}^{d}$. The corresponding system of matrix units $e_{i j}=\left|e_{i}\right\rangle\left\langle e_{j}\right|=e_{i} \otimes e_{j}^{*}$ identifies $\mathscr{B}\left(\mathscr{H}_{d}\right)$ with $\mathbb{M}_{d}(\mathbb{C})$. Then, for $A=\sum_{i, j=1}^{d} a_{i j} e_{i j}, B=\sum_{i, j=1}^{d} b_{i j} e_{i j}$ elements of $\mathcal{B}\left(\mathscr{H}_{d}\right)$, we define the Schur multiplication in $\mathcal{B}\left(\mathscr{H}_{d}\right)$ as usual,

$$
A \diamond B=\sum_{i, j=1}^{d} a_{i j} b_{i j} e_{i j},
$$

that is, componentwise,

$$
(A \diamond B)_{i j}:=a_{i j} b_{i j}
$$

Taking into account that

$$
(A \otimes B)_{(i, j)(k, l)}=a_{i k} b_{j l},
$$

we can extend the Schur multiplication to a map $m: \mathbb{M} \otimes \mathbb{M} \mapsto \mathbb{M}$ by putting

$$
m(X)_{i j}:=x_{(i, i)(j, j)} .
$$

It is a matter of routine to show that (2.6) defines a completely positive identitypreserving map from $\mathbb{M} \otimes \mathbb{M}$ onto $\mathbb{M}$.

${ }^{2}$ Equally well we could have taken $\mathbb{N}$ as the index-set without affecting most of the forthcoming analysis. 
Definition 1. A linear map $P: \mathbb{M} \mapsto \mathbb{M}$ is said to be Schur identity-preserving if its diagonal projection is the identity, i.e. if

$$
\mathbb{E}(P(I))=I .
$$

It is called an entangled Markov operator if, in addition,

$$
P(I) \neq I \text {. }
$$

If $P: \mathbb{M} \mapsto \mathbb{M}$ is Schur identity-preserving, then the map $\&: \mathbb{M} \otimes \mathbb{M} \mapsto \mathbb{M}$ given by

$$
\mathcal{E}=m \circ(\mathrm{id} \otimes P)
$$

is completely positive and identity-preserving, that is a transition expectation. The expectation $\&$ is called entangled if the corresponding Markov operator $P$ is entangled.

Starting from any transition expectation $\mathcal{E}: \mathbb{M} \otimes \mathbb{M} \mapsto \mathbb{M}$, a quantum Markov chain was constructed first in [1]. If one puts $\mathcal{E}_{A}(B):=\mathscr{E}(A \otimes B)$ and consider an "initial distribution" $\rho \in \delta(\mathbb{M})$ satisfying $\rho=\rho \circ \mathscr{E}_{I}$, a state $\omega \in \delta(\mathfrak{M})$ is uniquely determined by all "finite-dimensional distributions" as

$$
\omega\left(A_{1} \otimes \cdots \otimes A_{n}\right):=\rho\left(\varepsilon_{A_{1}} \circ \cdots \circ \mathcal{E}_{A_{n}}(I)\right) .
$$

Such a state is translation-invariant by construction, and is called $C^{*}$-finite correlated in [19], where transition expectations $\mathcal{E}: \mathbb{M} \otimes \mathbb{B} \mapsto \mathbb{B}$, for $\mathbb{B}$ another (in general different) finite-dimensional $C^{*}$-algebra are considered, generalizing the previous construction in [1]. We refer to the state on the UHF algebra generated by the triplet $(\mathbb{B}, \mathcal{E}, \rho)$ in the way explained above, as a quantum Markov chain. For further details, we refer to [1] and [10,19,20,36], and the references cited therein.

For the convenience of the reader, we report the following lemma which is useful in the following (see Lemma 2.5 of [20]).

Lemma 2.1. Let $s_{\rho}$ be the support of $\rho$ as above, and $p \geq s_{\rho}$ any projection. Then

$$
\rho \circ \mathcal{E}_{A_{1}} \circ \cdots \circ \mathcal{E}_{A_{n}}=\rho \circ \mathcal{E}_{A_{1}} \circ \cdots \circ \mathcal{E}_{A_{n}}(p \cdot p) .
$$

Proof. Let $q \leq s_{\rho}^{\perp}$ be a projection, and $A_{1}, \cdots, A_{n}$ positive elements of $\mathbb{M}$. We have, by the positivity of $\&$ and the invariance of $\rho$,

$$
\rho \circ \mathcal{E}_{A_{1}} \circ \cdots \circ \mathcal{E}_{A_{n}}(q) \leq\left(\prod_{j=1}^{n}\left\|A_{j}\right\|\right) \rho(q)=0 .
$$

Namely, by polarization, all functionals $\rho \circ \mathcal{E}_{A_{1}} \circ \cdots \circ \mathcal{E}_{A_{n}}$ are dominated by $\rho$ and we are done.

Definition 2. A quantum Markov chain is called entangled if the transition expectation $\&$ has the form (2.7), where $P$ is an entangled Markov operator. 
Notice that the present construction is a non-trivial generalization of the classical case where $\mathcal{E}(f \otimes g)=f \cdot P(g), P$ being a ordinary Markov operator, and the product in the r.h.s. is the usual pointwise product between functions.

The backward operator $\varepsilon_{I}$ of an entangled transition expectation has the property that

$$
\varepsilon_{I}(\mathbb{M}) \subset \mathbb{D}
$$

Conversely, we get for the forward operator $\mathcal{E}^{I}:=\mathscr{E}(\cdot \otimes I)$,

$$
\varepsilon^{I}(\mathbb{M}) \nsubseteq \mathbb{D}
$$

in the entangled case.

Let a classical Markov chain be described by a stochastic matrix $\Pi \in \mathbb{M}_{d}(\mathbb{C})$, together with a stationary distribution $\pi \in \mathbb{C}^{d}$. For $\Omega_{j} \equiv\{1, \ldots, d\}$ as in the previous section, construct the Markovian measure $\mu_{\Pi, \pi}$ on the space $\Omega=\prod_{j \in \mathbb{Z}} \Omega_{j}$ of all trajectories, see e.g. [22]. To this classical Markov chain we can associate a quantum Markov chain, that is a state $\varphi$ on $\mathfrak{M}$, by setting

$$
\varphi(A):=\int_{\Omega} \mathfrak{E}(A)(\omega) \mu_{\Pi, \pi}(\mathrm{d} \omega)
$$

where $\mathfrak{E}$ is the Umegaki conditional expectation of $\mathfrak{M}$ onto the Abelian algebra $\mathfrak{D}$. Such a diagonal lifting is quasi-classical, as it arises from a classical process in a trivial way. In the last situation, the involved Markov operator is an ordinary one.

Now, starting from a stochastic matrix, and a stationary distribution as above, we define a non-trivial quantum lifting we call entangled.

A large class of examples of entangled Markov operators can be produced following the scheme described below.

Definition 3. The entangled Markov operator associated to a stochastic matrix $\Pi$ and to the canonical systems of matrix units $\left\{e_{i j}\right\}_{i, j=1}^{d}$ of $\mathbb{M}$ is defined as

$$
P(A)_{i j}:=\sum_{k, l=1}^{d} \sqrt{\prod_{i k} \Pi_{j l}} a_{k l} .
$$

We can immediately verify that $P$ is a well-defined entangled Markov operator. Notice that one can use in (2.9) any "complex square root" instead of the positive one. Namely, if $\chi \in \mathbb{M}_{d}(\mathbb{T}), \mathbb{T}$ being the unit circle, then the map $P_{\chi}$ defined as

$$
P_{\chi}(A)_{i j}:=\sum_{k, l=1}^{d} \overline{\chi_{i k}} \chi_{j l} \sqrt{\Pi_{i k} \Pi_{j l}} a_{k l}
$$

gives rise to an entangled Markov operator as well.

We list some useful results relative to the transition expectation for the cases under considerations. 
Lemma 2.2. The transition expectation $\mathcal{E}=m \circ(\mathrm{id} \otimes P$ ), with $P$ as in (2.9), is generated by the isometry $V: \mathbb{C}^{d} \mapsto \mathbb{C}^{d} \otimes \mathbb{C}^{d}$ given by

$$
V e_{i}=\sum_{j=1}^{d} \sqrt{\Pi_{i j}} e_{i} \otimes e_{j},
$$

$\left\{e_{i}\right\}_{i=1}^{d}$ being the canonical basis of $\mathbb{C}^{d}$.

Proof. Immediately, take into account that

$$
V^{*} e_{i} \otimes e_{j}=\sqrt{\Pi_{i j}} e_{i}
$$

Notice that

$$
\begin{aligned}
\Pi & =p_{0} \Pi p_{0}+\sum_{\lambda \in \Lambda}\left(p_{0} \Pi p_{\lambda}+p_{\lambda} \Pi p_{\lambda}\right) \\
& =: p_{0} R_{0} p_{0}+\sum_{\lambda \in \Lambda}\left(p_{0} S_{\lambda} p_{\lambda}+p_{\lambda} \Pi_{\lambda} p_{\lambda}\right),
\end{aligned}
$$

and for each $\Pi_{\lambda}$,

$$
\Pi_{\lambda}=\sum_{j_{\lambda}=1}^{m_{\lambda}} p_{\lambda, j_{\lambda}} \Pi_{\lambda} p_{\lambda, j_{\lambda}+1}=: \sum_{j_{\lambda}=1}^{m_{\lambda}} p_{\lambda, j_{\lambda}} \Pi_{j_{\lambda}, j_{\lambda}+1} p_{\lambda, j_{\lambda}+1}
$$

(with the convention that $m_{\lambda}+1=1$ ) according to (6.1) and (6.2), respectively. If

$$
p:=\sum_{\lambda \in \Lambda} p_{\lambda}
$$

define $\tilde{\mathscr{E}}: \mathbb{M} \otimes \mathbb{M}_{p} \mapsto \mathbb{M}_{p}$ given by

$$
\tilde{E}:=p \mathcal{E} \Gamma_{\mathbb{M}_{p}}(\cdot) p
$$

Lemma 2.3. We have with the notations as above,

$$
\tilde{\mathcal{E}}\left(\mathbb{M} \otimes \mathbb{M}_{p_{\lambda, j_{\lambda}+1}}\right) \subset \mathbb{M}_{p_{\lambda, j_{\lambda}}}
$$

As a consequence,

$$
\tilde{E}\left(\mathbb{M} \otimes \mathbb{M}_{p_{\lambda}}\right) \subset \mathbb{M}_{p_{\lambda}}
$$

Proof. A straighforward computation, taking into account the definition of $\tilde{\mathscr{E}}$ and the canonical form of a stochastic matrix given in (6.1) and (6.2).

Let $\pi$ be a positive unit vector in $\mathbb{C}^{d}$, and construct the matrix $Q(\pi) \in \mathbb{M}_{d}(\mathbb{C})$ given by

$$
Q(\pi)_{i j}:=\sum_{k=1}^{d} \pi_{k} \sqrt{\Pi_{k i} \Pi_{k j}} .
$$

The matrix $Q(\pi)$ is positive by construction. Further, as $\Pi$ is stochastic, $Q(\pi)$ has unit trace, that is it defines a state on $\mathbb{M}_{d}(\mathbb{C})$. The map $Q$ defines a correspondence of the state space of $\mathbb{C}^{d}$ into the state space of $\mathbb{M}_{d}(\mathbb{C})$. 
Proposition 2.4. The map $Q$ given in (2.16) defines a one-to-one correspondence between the stationary distributions for $\Pi$ and stationary distributions for

$$
\mathcal{E}_{I} \equiv I \diamond P(\cdot),
$$

with $P$ as given in (2.9).

Proof. We start by proving that $Q(\pi)$ is stationary if $\pi$ is.

$$
\begin{aligned}
Q(\pi)\left(\mathcal{E}_{I}(A)\right) & \equiv \sum_{k, l, i, j} \pi_{k} \Pi_{k l} \sqrt{\Pi_{l i} \Pi_{l j}} a_{i j} \\
& =\sum_{l, i, j} \pi_{l} \sqrt{\Pi_{l i} \Pi_{l j}} a_{i j} \equiv Q(\pi)(A) .
\end{aligned}
$$

It is also injective as, if $A=\operatorname{diag}\left(a_{1}, \ldots, a_{d}\right)$, we get

$$
Q(\pi)(A) \equiv \sum_{k, l} \pi_{k} \Pi_{k l} a_{l}=\sum_{l} \pi_{l} a_{l} \equiv \pi(A) .
$$

Finally, the stationarity condition $\rho\left(\varepsilon_{I}\left(e_{i j}\right)\right)=\rho\left(e_{i j}\right) \equiv \rho_{i j}$ on the matrix units reads

$$
\rho_{i j}=\sum_{k} \rho_{k k} \sqrt{\Pi_{k i} \Pi_{k j}} \quad i, j=1, \ldots, d .
$$

Namely, $\rho$ is stationary iff it satisfies (2.17) which can be written as $\rho=$ $Q(\mathbb{E}(\rho))$. Namely, $Q$ is onto as $\mathbb{E}(\rho)$ is stationary on the diagonal algebra.

Notice that the map $Q_{\chi}$ associated to the entangled Markov operator in (2.10) is given by

$$
Q_{\chi}(\pi)_{i j}=\sum_{k=1}^{d} \pi_{k} \chi_{k i} \overline{\chi_{k j}} \sqrt{\Pi_{k i} \Pi_{k j}} .
$$

We are now ready to define an entangled lifting of a classical Markov chain determined by the stochastic matrix $\Pi$, and the stationary distribution $\pi$ for it. Indeed, it is the quantum Markov chain $\omega \in \delta(\mathfrak{M})$ generated by the triplet $(\mathbb{M}, \mathcal{E}, Q(\pi))$, where $\mathcal{E}: \mathbb{M} \otimes \mathbb{M} \mapsto \mathbb{M}$ is the transition expectation (2.7) constructed by the entangled Markov operator given in (2.9), and $Q(\pi)$ is the (unique) quantum stationary distribution associated to $\pi$ via (2.16). We refer to such a quantum Markov chain as an entangled Markov chain.

\section{Ergodic properties of entangled Markov chains}

Let $\omega \in \delta(\mathfrak{M})$ be the entangled Markov chain generated by the triplet $(\mathbb{M}, \mathcal{E}, Q(\pi))$ constucted in the previous section. We start by showing that $\omega$ has zero entropy density. In order to prove this, we begin with the following: 
Lemma 3.1. Let $M$ be a von Neumann algebra, $\varphi$ a normal state on it with projection-support $p$, and $T: M \mapsto M$ a completely positive identity-preserving normal map satisfying $\varphi \circ T=\varphi$. Then,

$$
p T(p) p=p .
$$

Proof. cf. [17], Theorem 2.16.

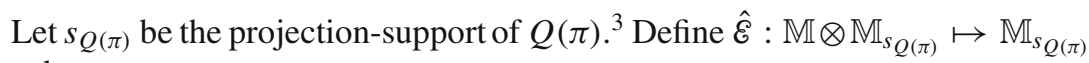
given by

$$
\hat{\mathcal{E}}:=s_{Q(\pi)} \mathcal{E}\left\lceil_{\mathbb{M}_{Q(\pi)}}(\cdot) s_{Q(\pi)} .\right.
$$

Proposition 3.2. The entangled Markov chain $\omega$ constucted as above has zero entropy density.

Proof. Put $q:=s_{Q(\pi)}$. By Lemma 2.1 , the triplet $\left(\mathbb{M}_{q}, \hat{\mathcal{E}}, Q(\pi) \Gamma_{\mathbb{M}_{q}}\right)$ is standard (using the terminology of [20]) and generates $\omega$. Further, Lemma 3.1 reads $q V^{*}(I \otimes q) V q=q$ when applied to the map $\varepsilon_{I}, V$ being the isometry generating $\&$ according to Lemma 2.2. We then conclude that $(I \otimes q) V q \Gamma_{q \mathbb{C}^{d}}$ is an isometry as well. Namely, $\omega$ is purely generated, according to Definition 1.1 of [20]. The assertion directly follows by [20, Theorem 1.4].

Let $\eta$ be the action of the completely reducible part $p \Pi p$ on the set of projections $\left\{\left\{p_{\lambda, j_{1}}, \ldots, p_{\lambda, j_{m_{\lambda}}}\right\}\right\}_{\lambda \in \Lambda}, p$ being given in (2.14). ${ }^{4}$ Such an action leaves each ergodic component $\left\{p_{\lambda, j_{\lambda}}\right\}_{\lambda=1}^{m \lambda}$ globally invariant, acting cyclically on it. Choose any projection, say $\bar{p}_{\lambda}:=p_{\lambda, \bar{j}}$, in each ergodic class $\lambda \in \Lambda_{\pi}$, where $\Lambda_{\pi}$ labels the ergodic components present in the stationary distribution $\pi$. Define for $\left\{A_{1}, \ldots, A_{n}\right\} \subset \mathbb{M}$,

$$
\begin{aligned}
& \varphi_{\lambda}\left(A_{1} \otimes \cdots \otimes A_{n}\right):=\pi\left(\bar{p}_{\lambda}\right)^{-1} Q(\pi)\left(\mathcal{E}_{A_{1}} \circ \cdots \circ \mathcal{E}_{A_{n}}\left(\eta^{-n} \bar{p}_{\lambda}\right)\right), \\
& \omega_{\lambda}\left(A_{1} \otimes \cdots \otimes A_{n}\right):=\pi\left(p_{\lambda}\right)^{-1} Q(\pi)\left(\mathcal{E}_{A_{1}} \circ \cdots \circ \mathcal{E}_{A_{n}}\left(p_{\lambda}\right)\right) .
\end{aligned}
$$

It can immediately be verified that

$$
\omega_{\lambda}=\frac{1}{m_{\lambda}} \sum_{k=1}^{m_{\lambda}} \varphi_{\lambda} \circ \tau^{k}
$$

where $\tau$ is the one-step shift on the chain.

The state $\varphi_{\lambda}$ describes the decomposition of $\omega$ into completely ergodic components, see e.g. [36] for the last definition. ${ }^{5}$

3 The projection-support of $Q(\pi)$ is in general smaller than that of $\pi$ as it is easily seen by considering the stochastic matrix $\left(\begin{array}{ll}\frac{1}{2} & \frac{1}{2} \\ \frac{1}{2} & \frac{1}{2}\end{array}\right)$.

4 Such an action is called in [20], the (classical) flow associated to $p \Pi p$.

5 The state $\varphi_{\lambda}$ is only $m_{\lambda}$-step translation invariant, $m_{\lambda}$ being the period of the component $\lambda$, and keeps track of the localization (modulo a period), see Section 5 for the precise way to define $\varphi_{\lambda}$ on $\mathfrak{M}$. 
Theorem 3.3. Let $(\Pi, \pi)$ consist of a stochastic matrix and a stationary distribution for it, and $\Lambda_{\pi}$ label the ergodic decomposition of $\pi$. Consider the entangled Markov chain $\omega$ on $\mathfrak{M}$ generated by the triplet $(\mathbb{M}, \mathcal{E}, Q(\pi))$ as above. Then

$$
\omega=\sum_{\lambda \in \Lambda_{\pi}} \frac{\pi\left(p_{\lambda}\right)}{m_{\lambda}} \sum_{k=1}^{m_{\lambda}} \varphi_{\lambda} \circ \tau^{k}
$$

describes the decomposition of $\omega$ into completely ergodic components.

Proof. It can immediately be verified that $\omega$ is given by (3.3). Further, the states appearing in the r.h.s. of (3.3) give rise to the decomposition into completely ergodic components of $\omega$, when restricted to the Abelian subalgebra $\mathfrak{D}$ of $\mathfrak{M}$. So, they are mutually different. It is then enough to show that the $\omega_{\lambda}$ are ergodic w.r.t. the one-step shift, and the $\varphi_{\lambda}$ are strongly clustering w.r.t. the $m_{\lambda}$-step shift, respectively.

Let $A=A_{1} \otimes \cdots \otimes A_{r}, B=B_{1} \otimes \cdots \otimes B_{s}$, we compute by applying Lemma 2.1,

$$
\begin{aligned}
& \frac{1}{n} \sum_{k=1}^{n} \omega_{\lambda}\left(A \tau^{k}(B)\right)=\pi\left(p_{\lambda}\right)^{-1} \\
& \quad \times Q(\pi)\left(\varepsilon_{A_{1}} \circ \cdots \circ \varepsilon_{A_{r}} \circ\left(\frac{1}{n} \sum_{k=0}^{n-1} \varepsilon_{I}^{k}\right)\left(\varepsilon_{I} \circ \tilde{\mathcal{E}}_{B_{1}} \circ \cdots \circ \tilde{\mathcal{E}}_{B_{s}}\left(p_{\lambda}\right)\right)\right),
\end{aligned}
$$

where $\tilde{\mathcal{E}}$ is given by (2.15). Put $D:=\mathcal{E}_{I} \circ \tilde{\mathcal{E}}_{B_{1}} \circ \cdots \circ \tilde{\mathcal{E}}_{B_{s}}\left(p_{\lambda}\right)$. By Lemma 2.3 and (2.8), we argue that $D \in \mathbb{D}_{p_{\lambda}}$. As a consequence, we have, by (6.5),

$$
\lim _{n} \frac{1}{n} \sum_{k=0}^{n-1} \varepsilon_{I}^{k}(D)=\lim _{n} \frac{1}{n} \sum_{k=0}^{n-1} \Pi_{\lambda}^{k} D=Q(\pi)\left(\mathcal{E}_{B_{1}} \circ \cdots \circ \mathcal{E}_{B_{s}}\left(p_{\lambda}\right)\right) p_{\lambda} .
$$

Collecting these together, we get

$$
\begin{aligned}
\lim _{n} \frac{1}{n} \sum_{k=1}^{n} \omega_{\lambda}\left(A \tau^{k}(B)\right)= & Q(\pi)\left(\varepsilon_{A_{1}} \circ \cdots \circ \mathcal{E}_{A_{r}}\left(p_{\lambda}\right)\right) \\
& \times Q(\pi)\left(\mathcal{E}_{B_{1}} \circ \cdots \circ \mathcal{E}_{B_{s}}\left(p_{\lambda}\right)\right) \equiv \omega_{\lambda}(A) \omega_{\lambda}(B) .
\end{aligned}
$$

Namely, $\omega_{\lambda}$ is ergodic (see e.g. [23, Theorem 2.1]). The mixing property w.r.t. the $m_{\lambda}$-step shift for the $\varphi_{\lambda}$ is proven in the same way, taking into account (6.3), (6.4) and the proof follows.

As an immediate consequence, we get:

Theorem 3.4. The following assertions hold true under the assumptions of Theorem 3.3:

(i) the state $\omega$ is ergodic w.r.t the spatial translation iff the set $\Lambda_{\pi}$ is a singleton;

(ii) the state $\omega$ is strongly clustering w.r.t the spatial translation iff the set $\Lambda_{\pi}$ is a singleton, and the corresponding block in the decomposition (2.12) of $\Pi$ is primitive.

Further, if (ii) is satisfied, then the state $\omega$ is pure. 
Proof. (i) and (ii) immediately follow from the previous theorem. Further, (ii) means that $\omega$ is strongly clustering. By Proposition 3.2, $\omega$ has vanishing mean entropy. The proof follows by (ii) of Theorem 1.5 of [20].

\section{Some remarks}

We start by discussing the structure of the support of the stationary distribution $\pi$ and the corresponding quantum lifting $Q(\pi)$ which are in general different, see footnote 3 .

Let $\pi$ be a stationary distribution with support $s_{\pi}$. Then, taking into account the structure of the stochastic matrix $\Pi$ (see Section 6), we have

$$
s_{\pi}=\sum_{\lambda \in \Lambda_{\pi}} p_{\lambda},
$$

where $p_{\lambda}$ is the projection corresponding to the ergodic component $\lambda$, and $\Lambda_{\pi}$ is the set of the ergodic classes associated to the stationary distribution $\pi$. Looking at each ergodic component $\lambda$, we obtain for the corresponding projection $p_{\lambda}$,

$$
p_{\lambda}=\sum_{j=1}^{m_{\lambda}} p_{\lambda, j}
$$

where $m_{\lambda}$ is the period of the ergodic component $\lambda$, and the $p_{\lambda, j}$ describe the decomposition of the $m_{\lambda}$-th power $\left(p_{\lambda} \Pi p_{\lambda}\right)^{m_{\lambda}}$ of the irreducible component $p_{\lambda} \Pi p_{\lambda}$ into primitive pieces, see (2.12) and (2.13).

Taking into account (2.17), together with the structure of a completely reducible stochastic matrix, we conclude that there are nontrivial subprojections $q_{\lambda, j_{\lambda}}$ with $0<q_{\lambda, j_{\lambda}} \leq p_{\lambda, j_{\lambda}}$, such that

$$
s_{Q(\pi)}=\sum_{\lambda \in \Lambda_{\pi}}\left(\sum_{j_{\lambda}=1}^{m_{\lambda}} q_{\lambda, j_{\lambda}}\right) .
$$

In the previous section we have shown that the structure of the peripheral part of the spectrum of $\Pi$ completely describes the ergodic properties of the corresponding entangled chain (see Theorem 3.3 and Theorem 3.4). It could be of interest to understand the structure of the peripheral part of the spectrum of the quantum counterpart $\hat{\mathcal{E}}_{I}$ given by (3.1).

According to [20], we consider $\mathbb{Z}_{1}, \mathfrak{Z}$ which are the eigenspaces corresponding to the eigenvalue 1 , and the span of the eigenvectors corresponding to the peripheral spectrum of $\hat{\mathscr{E}}_{I}$, respectively. The corresponding classical algebras $\mathfrak{Z}_{1, c l}, \mathbb{Z}_{c l}$ are spanned by the minimal projections $\left\{p_{\lambda}\right\}_{\lambda \in \Lambda_{\pi}}$ and $\left\{\left\{p_{\lambda, j_{1}}, \ldots, p_{\lambda, j_{m_{\lambda}}}\right\}\right\}_{\lambda \in \Lambda_{\pi}}$, respectively.

By using the standard properties of completely positive maps between matrix algebras $([15,16])$, it is straightforward to show that $\mathcal{Z}_{1, c l}, \mathcal{Z}_{c l}$ are naturally embedded in the corresponding quantum Abelian algebras $\mathcal{Z}_{1}, \mathfrak{Z}$, where the embedding is given by

$$
\mathfrak{Z}_{c l} \longrightarrow\left(\mathcal{Z}_{c l}\right)_{s_{Q(\pi)}} \subset \mathcal{Z}
$$


We conjecture that the above embedding is indeed an isomorphism.

Another connected question concerns the minimal generating triplet for $\omega$ (see [20, Definition 1.2]).

Define

$$
\mathbb{B}:=\bigoplus_{\lambda \in \Lambda_{\pi}}\left(\bigoplus_{j_{\lambda}=1}^{m_{\lambda}} \mathbb{M}_{q_{\lambda, j_{\lambda}}}\right), \quad \mathcal{F}:=\hat{\mathcal{E}}\left\lceil_{\mathbb{B}}, \quad \rho:=Q(\pi) \Gamma_{\mathbb{B}} .\right.
$$

It is easily shown that the entangled Markov chain $\omega$ under consideration is generated by the standard triplet $(\mathbb{B}, \mathcal{F}, \rho)$ as well. We also conjecture that the triplet $(\mathbb{B}, \mathcal{F}, \rho)$ is minimal.

We are not able to prove or disprove the above conjectures in general. ${ }^{6}$ However, they are both true in the case when $s_{\pi}=s_{Q(\pi)}$. We verify the second assertion, the first one being trivial by (2.8).

In the last situation, there exists, after reordering the indices, a power $\left(s_{\pi} \Pi s_{\pi}\right)^{m}$ made of diagonal blocks of primitive matrices, $m$ being a common multiple of all periods $\left\{m_{\lambda}\right\}_{\lambda \in \Lambda_{\pi}}$. Taking into account the definition of a primitive matrix, there exists an integer $l$ such that $\left(s_{\pi} \Pi s_{\pi}\right)^{m l}$ is made of diagonal blocks (the same as the previous ones) of strictly positive matrices. Notice that the projections relative to such diagonal blocks are precisely the identities of the corresponding summands of $\mathbb{B}$. Put $n=m l$. It is then enough to show that for each fixed pair $i, j$ in the set of indices for which general elements of $\mathbb{B}$ have non-zero entries, there exist $A_{1}, \ldots, A_{n} \in \mathbb{M}$ such that

$$
\varepsilon_{A_{1}} \circ \cdots \circ \mathcal{E}_{A_{n}}(I)_{k l}=a \delta_{k, i} \delta_{l, j},
$$

for some $a>0$. We choose $A_{1}=e_{i j}, A_{2}=\cdots=A_{n}=E$, where $E$, the matrix with all entries consisting of 1 , is the unity for the Schur multiplication. The assertion follows as for the set of involved indices,

$$
\varepsilon_{e_{i j}} \circ \varepsilon_{E} \circ \cdots \circ \varepsilon_{E}(I)_{i j} \geq \sqrt{\sum_{k} \Pi_{i k}^{n} \Pi_{j k}^{n}}>0 .
$$

Finally, we remark that the disintegraton (3.3) of the entangled lifting of a Markov chain leads to a convex combination of pure states which are again entangled Markov chains. Namely, let $\varphi_{\lambda}$ given in (3.2) be fixed. Then, on the regrouped algebra

$$
\bar{\bigotimes}_{\mathbb{Z}}(\underbrace{\mathbb{M} \otimes \cdots \otimes \mathbb{M}}_{m_{\lambda} \text {-times }})
$$

the state $\varphi_{\lambda}$ is generated by the isometry

$$
W:=(I \otimes \cdots \otimes I \otimes V) \cdots(I \otimes I \otimes V)(I \otimes V)
$$

( $V$ as in (2.11)), and has support $q_{\lambda, \bar{j}}$. Here, $q_{\lambda, \bar{j}}$ is the projection appearing in (4.1) such that $q_{\lambda, \bar{j}} \leq p_{\lambda, \bar{j}}$, for $p_{\lambda, \bar{j}} \equiv \bar{p}_{\lambda}$ given in (3.2). The assertion follows again by Theorem 1.5 of [20].

6 As the ergodic properties of the entangled Markov chains are determined by the structure of the underlying stochastic matrices, this does not affect our analysis. 


\section{On non-homogeneous entangled Markov chains}

In view of applications to the multi-dimensional case (see [4, Section 2], [5]), we study in the non-homogeneous case, the basic properties emerging from the construction in Section 2. In this situation,

$$
\mathfrak{M}:=\bar{\bigotimes}_{j \in \mathbb{Z}} \mathbb{M}_{d_{j}}(\mathbb{C}){ }^{*},
$$

where $d_{j}$ depends possibly on the place $j$.

Consider a sequence $\left\{\mathcal{E}^{j}\right\}_{j \in \mathbb{Z}}$ of transition expectations

$$
\mathscr{E}^{j}: \mathbb{M}_{d_{j}}(\mathbb{C}) \otimes \mathbb{M}_{d_{j+1}} \mapsto \mathbb{M}_{d_{j}}(\mathbb{C})
$$

(i.e. completely positive, identity-preserving maps). Consider also a suitable sequence of states $\rho(j)$ on $\mathbb{M}_{d_{j}}(\mathbb{C})$ satisfying the compatibility conditions

$$
\rho(j) \circ \varepsilon_{I}^{j}=\rho(j+1), \quad j \in \mathbb{Z} .
$$

The sequences $\left\{\mathscr{E}^{j}\right\}_{j \in \mathbb{Z}},\{\rho(j)\}_{j \in \mathbb{Z}}$ generate a state $\omega$ on $\mathfrak{M}$ called non-homogeneous quantum Markov chain, and characterized by the property that on elements $A_{j} \otimes \cdots \otimes A_{l}$, localized in the region $\mathfrak{M}_{\{j, \ldots, l\}}$, it has the form

$$
\omega\left(A_{j} \otimes \cdots \otimes A_{l}\right):=\rho(j)\left(\mathcal{E}_{A_{j}}^{j} \circ \cdots \circ \varepsilon_{A_{l}}^{l}(I)\right) .
$$

For a general non-homogeneous Markov chain, the first step is to exhibit a sequence of states satisfying (5.1).

Proposition 5.1. The set of non-homogeneous entangled Markov chains on $\mathfrak{M}$ is non-void.

Proof. We have to prove the existence of a map $\rho: \mathbb{Z} \mapsto \prod_{j \in \mathbb{Z}} \&\left(\mathbb{M}_{d_{j}}(\mathbb{C})\right)$ with $\rho(j) \in \delta\left(\mathbb{M}_{d_{j}}(\mathbb{C})\right)$ satisfying (5.1). We start by choosing a sequence of maps $\left\{\rho_{k}\right\}_{k \in \mathbb{Z}_{-}}$such that each $\rho_{k}$ satisfies (5.1) for all $j>k$. By compactness there exists a pointwise convergent subsequence $\rho$. For each fixed $j \in \mathbb{Z}$, we get

$$
\rho(j) \circ \mathcal{E}_{I}^{j}=\lim _{n} \rho_{k_{n}}(j) \circ \varepsilon_{I}^{j}=\lim _{n} \rho_{k_{n}}(j+1)=\rho(j+1),
$$

which is the assertion.

In order to obtain non-homogeneous examples of entangled Markov chains, we can start with a sequence $\left\{P_{j}\right\}_{j \in \mathbb{Z}}$ of entangled Markov operators $P_{j}: \mathbb{M}_{d_{j+1}}(\mathbb{C}) \mapsto$ $\mathbb{M}_{d_{j}}(\mathbb{C})$ and construct the sequence $\left\{\mathscr{E}^{j}\right\}_{j \in \mathbb{Z}}$ of transition operators by putting

$$
\mathcal{E}^{j}:=m_{j} \circ\left(\mathrm{id} \otimes P_{j}\right),
$$

$m_{j}$ being the Schur multiplication on $\mathbb{M}_{d_{j}}(\mathbb{C})$.

In order to obtain quantum liftings of non-homogeneous classical Markov

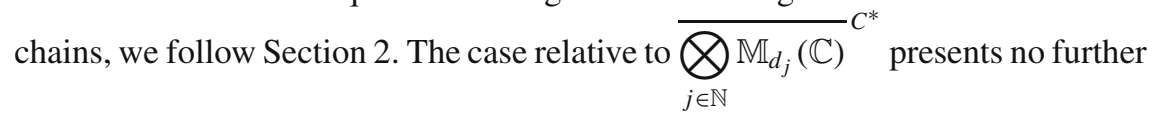
complications. Proposition 5.1 assures that one can construct non-trivial examples

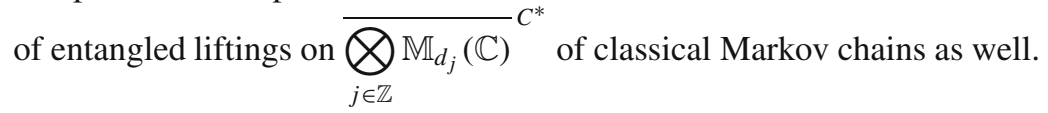




\section{Appendix}

We collect some standard properties of stochastic matrices repeatedly used in the sequel. The reader is referred to Section I.9 of [37] for further details.

A stochastic matrix $\Pi \in \mathbb{M}_{d}(\mathbb{C})$ is said to be irreducible if there exists no permutation $P$ such that

$$
Р П P^{-1}=\left(\begin{array}{cc}
A & B \\
0 & C
\end{array}\right) .
$$

A stochastic matrix $\Pi$ is said to be primitive if there exists $l \geq 1$ such that $\left(\Pi^{l}\right)_{i j}>0$ for all indices $i, j$.

Let the stochastic matrix $\Pi$ be given. It can be written, after a possible reordering of indices, as

$$
\Pi=\left(\begin{array}{cccc}
R_{0} & S_{1} & \cdots & S_{|\Lambda|} \\
0 & \Pi_{1} & \cdots & 0 \\
\cdot & \cdot & \cdots & \cdot \\
0 & 0 & \cdots & \Pi_{|\Lambda|}
\end{array}\right)
$$

where the $S_{\lambda}$ are not all zero and the $\Pi_{\lambda}$ irreducible and stochastic.

Here, $\Lambda:=(1, \ldots,|\Lambda|)$ labels the ergodic classes corresponding to the stochastic submatrices $\left\{\Pi_{\lambda}\right\}_{\lambda \in \Lambda}$. The zero row in (6.1) can be empty (this happens if and only if there is no transient "states" for the classical chain), in which case the matrix $\Pi$ is said to be completely reducible.

Each irreducible submatrix $\Pi_{\lambda}$ has a unique stationary distribution described by a row vector $x_{\lambda}$. Further, each stationary distribution $x$ for a stochastic matrix $\Pi$ given by (6.1), is a convex combination of the stationary distributions of its ergodic components,

$$
x=\left(0, \alpha_{1} x_{1}, \ldots, \alpha_{|\Lambda|} x_{|\Lambda|}\right) ; \quad \sum_{\lambda=1}^{|\Lambda|} \alpha_{\lambda}=1 ; \alpha_{\lambda} \geq 0, \lambda=1, \ldots,|\Lambda| .
$$

Consider a primitive stochastic matrix $\Pi$. Then its $k$-th powers $\Pi^{k}$ converge to a rank-one stochastic projection, that is

\[ \lim _{k} \Pi^{k}=(\cdot, x) e \]
where $x$ is the unique stationary distribution for $\Pi$, and $e \equiv\left(\begin{array}{c}1 \\ \vdots \\ 1\end{array}\right)$ is the identity of $\mathbb{C}^{d}$ (or equivalently the common identity $I$ of $\mathbb{D} \subset \mathbb{M}$ ).

Now let the stochastic matrix $\Pi$ be irreducible but not primitive. It can be written, after a possible reordering of indices, as

$$
\Pi=\left(\begin{array}{ccccc}
0 & \Pi_{1,2} & 0 & \cdots & 0 \\
0 & 0 & \Pi_{2,3} & \cdots & 0 \\
\cdot & \cdot & \cdot & \cdots & \cdot \\
0 & 0 & 0 & \cdots & \Pi_{m-1, m} \\
\Pi_{m, 1} & 0 & 0 & \cdots & 0
\end{array}\right),
$$

where $m>1$ is the index of imprimitivity (or the period) of $\Pi$. 
It is well known that

$$
\Pi^{m}=\left(\begin{array}{cccc}
\Pi_{1}^{(m)} & 0 & \cdots & 0 \\
0 & \Pi_{2}^{(m)} & \cdots & 0 \\
\cdot & \cdot & \cdots & \cdot \\
0 & 0 & \cdots & \Pi_{m}^{(m)}
\end{array}\right),
$$

where the $\Pi_{j}^{(m)}$ are primitive. Further, we have for the $k$-th powers $\Pi^{k m}$ of $\Pi^{m}$,

$$
\lim _{k} \Pi^{k m}=\left(\begin{array}{cccc}
Q_{1}^{(m)} & 0 & \cdots & 0 \\
0 & Q_{2}^{(m)} & \cdots & 0 \\
\cdot & \cdot & \cdots & \cdot \\
0 & 0 & \cdots & Q_{m}^{(m)}
\end{array}\right) .
$$

Here, the $Q_{j}^{m}$ are stochastic rank-one projections given by

$$
Q_{j}^{(m)}=\left(\cdot, x_{j}^{(m)}\right) p_{j}
$$

where $x_{j}^{(m)}$ is the vector describing the unique stationary distribution for $\Pi_{j}^{(m)}$ and $p_{j}$ is its support as a projection of $\mathbb{C}^{d}$. It follows that the ergodic limit of the irreducible matrix $\Pi$ exists and is a rank-one stochastic projection,

$$
\lim _{n} \frac{1}{n} \sum_{k=0}^{n-1} \Pi^{k}=(\cdot, x) e,
$$

where $x$ is the unique stationary distribution for $\Pi$, and $e$ is as above.

Acknowledgements. The second-named author (F. F.) is grateful to P. Baldi and G. Scalia Tomba for some fruitful discussions.

\section{References}

1. Accardi, L.: Non commutative Markov chains. In: Proceedings of Internat. School of Math. Phys., pp. 268-295. Camerino 1974

2. Accardi, L.: Topics in quantum probability. Phys. Rep. 77, 169-192 (1981)

3. Accardi, L.: Some trends and problems in quantum probability. In: Quantum probability and applications to the quantum theory of irreversible processes. ed. by L. Accardi, A. Frigerio, V. Gorini. Lect. Notes Math., vol. 1055, pp. 1-19. Berlin: Springer 1984

4. Accardi, L., Fidaleo, F.: Non homogeneous quantum Markov states and quantum Markov fields. J. Funct. Anal. 200, 324-347 (2003)

5. Accardi, L., Fidaleo, F.: Quantum Markov fields. Infin. Dimens. Anal. Quantum Probab. Relat. Top. 6, 123-138 (2003)

6. Accardi, L., Watson, G.S.: Quantum random walks and coherent quantum chains. In: Quantum Probability and Applications IV. Lect. Notes Math., vol. 1396, pp. 73-88. Berlin: Springer 1987

7. Aharonov, D., Ambainis, A., Kempe, J., Vazirani, U.V.: Quantum walks on graphs. In: Proceedings of the 33rd Annual ACM Symposium on Theory of Computing, pp. 50-59. New York: ACM Press 2001 
8. Ambainis, A., Bach, E., Nayak, A., Vishwanath, A., Watrous, J.: One-dimensional quantum walks. In: Proceedings of the 33rd Annual ACM Symposium on Theory of Computing, pp. 37-49. New York: ACM Press 2001

9. Bach, E., Coppersmith, S., Goldschen, M.P., Joynt, R., Watrous, J.: One-dimensional quantum walks with absorption boundaries. quant-ph/0207008 (2002)

10. Bratteli, O., Jorgensen, P.E.T., Kishimoto, A., Werner, R.F.: Pure states on $\mathcal{O}_{d}$. J. Oper. Theory 43, 97-143 (2000)

11. Brun, T.A., Carteret, H.A.: The quantum to classical transition for random walk. quant$\mathrm{ph} / 0208195$ (2002)

12. Brun, T.A., Carteret, H.A., Ambainis, A.: Quantum walks driven by many coins. Phys. Rev. A 67 (2003), 052317, 17 pp.

13. Brun, T.A., Carteret, H.A., Ambainis, A.: Quantum random walks with decoherent coins. Phys. Rev. A 67 (2003), 032304, 9 pp.

14. Childs, A.M., Farhi, E., Gutmann, S.: An example of the difference between quantum and classical random walks. Quantum Inf. Process. 1, 35-43 (2002)

15. Choi, M.-D.: Completely positive maps on complex matrices. Linear Algebra Appl. 10, 285-290 (1975)

16. Fagnola F., Bidot R.P.: Irreducible and periodic positive maps. Preprint 2003

17. Fagnola, F., Rebolledo, R.: Lectures in qualitative analysis of quantum Markov semigroups. In: Quantum Probability and White Noise Analysis, vol XIV, ed. by L. Accardi, F. Fagnola. River Edge: World Scientific 2002

18. Fannes, M., Nachtergaele, B., Slegers, L.: Functions of Markov processes and Markov measures. Rev. Math. Phys. 4, 39-64 (1992)

19. Fannes, M., Nachtergaele, B., Werner, R.F.: Finitely correlated states of quantum spin chains. Commun. Math. Phys. 144, 443-490 (1992)

20. Fannes, M., Nachtergaele, B., Werner, R.F.: Finitely correlated pure states. J. Funct. Anal. 120, 511-534 (1994)

21. Fidaleo, F.: Infinite dimensional entangled Markov chains. Preprint 2004

22. Guikhman, I., Skorokhod, A.: Introduction à la théorie des processus aléatoires. Moscow: MIR 1980

23. Kastler, D.: Equilibrium states of matter and operator algebras. In: Symposia mathematica, vol. XX, pp. 49-107. London: Academic Press 1976

24. Kendon, V., Tregenna, B.: Decoherence is useful in quantum walks. quant-ph/0209005 (2002)

25. Kendon, V., Tregenna, B.: Decoherence in a quantum walk on the line. quant-ph/0210047 (2002b)

26. Konno, N.: Quantum random walks in one dimension. Quantum Inf. Process. 1, 345-354 (2003)

27. Konno, N.: A new type of limit theorems for the one-dimensional quantum random walk. quant-ph/0206103 (2002)

28. Konno, N.: Limit theorems and absorpion problems for quantum random walks in one dimension. Quantum Inf. Comput. 2, Suppl., 578-595 (2002)

29. Konno, N., Namiki, T., Soshi, T.: Symmetry of distribution for the one-dimensional Hadamard walk. Interdiscip. Inf. Sci. 10, 11-22 (2004)

30. Konno, N., Namiki, T., Soshi, T., Sudbury, A.: Absorption problems for quantum random walks in one dimension. J. Phys. A 36, 241-253 (2003)

31. Leroux P.: Coassociative grammar, periodic orbits and quantum random walk over $\mathbb{Z}^{1}$. quant-ph/0209100 (2002)

32. Mackay, T.D., Bartlett, S.D., Stephanson, L.T., Sanders, B.C.: Quantum walks in higher dimensions. J. Phys. A 35, 2747-2753 (2002)

33. Meyer, D.A.: From quantum cellular automata to quantum lattice gases. J. Stat. Phys. 85, 551-574 (1996)

34. Moore, C., Russell, A.: Quantum walks on the hypercubes. quant-ph/0104137 (2001)

35. Nachtergaele, B.: The spectral gap for some spin chains with discrete symmetry breaking. Commun. Math. Phys. 175, 565-606 (1992) 
36. Nachtergaele, B.: Quantum Markov chains: applications to physics. Centro V. Volterra, preprint n. 497 (2001)

37. Schaefer, H.H.: Banach Lattices and positive operators. Berlin, Heidelberg, New York: Springer 1974

38. Severini, S.: Line diagrams and line quantum random walks. Preprint quant-ph/0210055 (2002)

39. Travaglione, B.C., Milburn, G.J.: Implementing the quantum random walk. Phys. Rev. A 65 (2002), 032310, 5 pp.

40. Yamasaki, T., Kobayashi, H., Imai, H.: An analysis of absorbing times of quantum walks. Lect. Notes Comput. Sci., vol. 2509, pp. 315-329. Berlin: Springer 2002 